\title{
The Effect of Drying Rate on Shrinkage of Alkali-Activated Slag Cements
}

\author{
Hailong Ye, Christopher Cartwright, Farshad Rajabipour, Aleksandra Radlińska \\ Department of Civil and Environmental Engineering, The Pennsylvania State University, State College, \\ PA, 16801, United States
}

\begin{abstract}
The volumetric instability of alkali-activated slag (AAS) binders has raised concerns and impeded the acceptance of this Portland cement-free material. The objective of present work is to characterize the influence of drying rate on drying shrinkage behavior of alkali-activated slag mortars to better understand the mechanisms responsible for its large shrinkage deformation. A series of four AAS mortar mixtures with varying activator composition, as well as a reference Portland cement mortar was cast and dried at different relative humidities, i.e. $30 \%, 50 \%, 70 \%$ and $85 \% \mathrm{RH}$. Drying took place inside nitrogen purged environmental chambers, for the purpose of eliminating the contribution of carbonation to the total volumetric change of AAS. The shrinkage and corresponding mass loss of $1.27 \mathrm{~cm} \times 1.27 \mathrm{~cm} \times 12.7 \mathrm{~cm}$ prisms were measured as a function of time. The results show that shrinkage of AAS varies largely depending on the drying rate, i.e. ambient $\mathrm{RH}$. Interestingly, even though the drying mass loss increases with reducing the $\mathrm{RH}$, the magnitude of shrinkage is the largest for samples stored at $50 \%$ and $70 \% \mathrm{RH}$, depending on the mixture type. Possible causes of these irregular behaviors are discussed. It is concluded that the drying rate has a much more significant influence on AAS than on OPC, which implies a more complicated shrinkage mechanism for AAS samples stored at various relative humidities.
\end{abstract}

\section{INTRODUCTION}

Ground-granulated blast furnace slag (GGBFS) is an amorphous by-product of the steel industry. It has a latent hydraulic reactivity, which can be catalyzed by proper activators to form cementitious materials. Alkali-activated slag (AAS) is one type of alkali-activated cement products which can offer superior properties over ordinary Portland cement (OPC) concrete, including high compressive strength at early age and excellent durability against chemical (acid, sulfate) attack (Shi et al., 2005). In addition, AAS has lower $\mathrm{CO}_{2}$ emissions than OPC, and its ecofriendly advantage mainly originates from utilization of by-products (Jiang et al., 2014). However, some drawbacks of AAS still exist which hinder its broad acceptation and industry application. One of the main disadvantages for potential applications of AAS in construction industry is the volumetric instability, i.e. high shrinkage of AAS (Cartwright et al., 2013a, 2014; Shi et al., 2005).

Many researchers have reported that AAS exhibits significantly higher drying shrinkage than that of OPC (Bakharev et al., 2000; Bakharev et al., 1999; Cartwright et al., 2013a, 2014; Cartwright et al., 2013b; Collins and Sanjayan, 2000; Melo Neto et al., 2008; Shi et al., 2005). For example, Cartwright et al. measured the drying shrinkage of AAS mortar of four mixtures under $50 \% \mathrm{RH}$, documenting that AAS has significantly higher drying shrinkage, finer pore structure, and lower stiffness than OPC (Cartwright et al., 2014). Collins et al. also observed the high shrinkage of AAS and related this phenomena to uniform pore size distribution in AAS (Collins and Sanjayan, 2000). Similar experimental results and conclusions were also reported by Neto et al., who evaluated the shrinkage of AAS with different amounts of dissolved silica from activators (Melo Neto et al., 2008). However, the scientific reasoning behind the high drying shrinkage behavior of AAS is still unknown. Some authors hypothesize that upon drying, a high capillary force forms in the materials of fine pore structure like AAS (Collins and Sanjayan, 2000; Melo Neto et al., 2008). This assumption has not been verified though, as drying shrinkage tests under a broad range of relative humilities (e.g. from $30 \% \mathrm{RH}$ to $100 \%$ $\mathrm{RH}$ ) have not yet been conducted. Additionally, 
previous research merely observes the drying shrinkage of AAS under a constant relative humidity (50\% RH in most cases). As such, this limited availability of experimental data prevents a more thorough understanding of the shrinkage behavior of AAS.

This paper presents the advanced drying shrinkage characterization of AAS mortar with various natures and dosages of activators. In particular, drying shrinkage of AAS is measured under two types of drying conditions, namely rapid drying and step-wise drying, as explained later. Both of these drying conditions cover a broad range of relative humidities from $30 \%$ to $85 \% \mathrm{RH}$. The objective of present work is to contribute to the better understanding of drying shrinkage behavior of AAS and its associated underlying mechanism. The outcomes of this research can provide fundamental information on the shrinkage mechanism of AAS, and provide further insights into possible shrinkage reduction and mitigations strategies.

\section{EXPERIMENTAL PROCEDURE}

\subsection{Materials}

A grade 120 (according to ASTM C989/C989M13) ground granulated blast-furnace slag with a density of $2.89 \mathrm{~g} / \mathrm{cm}^{3}$ and a Type I Portland cement (according to ASTM C150/C150M-12) with a density of $3.14 \mathrm{~g} / \mathrm{cm}^{3}$ were used in present study. Table 1 shows the oxide composition of the aforementioned materials. When preparing the activating solutions, sodium hydroxide, sodium silicate solution, and distilled water were used. The sodium silicate solution $\left(\mathrm{Na}_{2} \mathrm{O}\left(\mathrm{SiO}_{2}\right)_{1.6}\left(\mathrm{H}_{2} \mathrm{O}\right)_{10}\right)$ had a specific gravity of 1.60 and $\mathrm{pH}$ of 13.7 at $20^{\circ} \mathrm{C}$. The $\mathrm{NaOH}$ pellets had a specific gravity of 2.13 . To prepare the activating solutions, reagent $\mathrm{NaOH}$ pellets were dissolved in water and covered in closed beaker to avoid the loss of moisture and ingress of external $\mathrm{CO}_{2}$. The solution was then shaken for 5 minutes and set aside for approximately two hours to allow for heat dissipation. In addition, a small dosage of water-reducing admixture (WRA) was added to AAS systems to ensure adequate workability. A natural river sand (according to ASTM C33/C33M-13) was also used for each mixture. The sand had a fineness modulus of 2.60 , oven dry specific gravity of 2.52 , and absorption capacity of $2.0 \%$.
Four AAS mortars and a control OPC mortar were designed using a constant volumetric liquid (water+activator) to solid (slag or cement) ratio of 1.30 . This resulted in an initial binder porosity of $56.5 \%$ in all five mixtures. For the control OPC mixture, the mass-based w/c was 0.414 . For the AAS mixtures, the mass-based liquid to solid ratio was in the range 0.489 to 0.510 , depending on the concentration and density of the activating solutions. The details of mixture proportions are shown in Table 2 and Table 3. In particular, mortars AAS1 (lower silica concentration) and AAS2 (higher silica concentration) were activated using an alkaline solution which contained a combination of dissolved sodium hydroxide $(\mathrm{NaOH})$ pellets, aqueous sodium silicate $\left(\mathrm{Na}_{2} \mathrm{O}\left(\mathrm{SiO}_{2}\right)_{1.6} \cdot 10 \mathrm{H}_{2} \mathrm{O}\right)$ (waterglass), and water. Mortars AAS3 and AAS4 were activated solely by $\mathrm{NaOH}$ in water (either $2 \mathrm{M}$ or $4 \mathrm{M}$ ). It should be mentioned here that the amount of water reported in Table 2 accounts for the absorption of oven-dried sand.

Table 1. Oxide compositions of slag and Portland cement (mass \%)

\begin{tabular}{lccccccccc}
\hline C & S & A & $\mathbf{M}$ & $\underline{\mathbf{S}}$ & $\mathbf{F}$ & $\mathbf{N}$ & $\mathbf{K}$ & LOI \\
\hline Slag & 47.5 & 30.8 & 11.45 & 3.65 & 3.03 & 1.81 & 0.17 & 0.38 & 1.17 \\
Cement 62.5 & 19.9 & 5.44 & 2.31 & 4.93 & 2.26 & 0.30 & 0.89 & 0.86 \\
\hline Notes: Following abbreviated symbols are used above \\
$\mathrm{C}=\mathrm{CaO}, \mathrm{S}=\mathrm{SiO}_{2}, \mathrm{~A}=\mathrm{Al}_{2} \mathrm{O}_{3}, \mathrm{M}=\mathrm{MgO}, \mathrm{F}=$ & $\mathrm{Fe}_{2} \mathrm{O}_{3}, \underline{\mathrm{S}}=\mathrm{SO}_{3}$, \\
$\mathrm{H}=\mathrm{H}_{2} \mathrm{O}, \mathrm{N}=\mathrm{Na}_{2} \mathrm{O}, \mathrm{K}=\mathrm{K}_{2} \mathrm{O}$.
\end{tabular}

Table 2. Mortar mixture proportions (in grams per liter of mortar)

\begin{tabular}{cccccccc}
\hline Mix ID & $\mathrm{NaOH} \mathrm{Na}_{2} \mathrm{O}\left(\mathrm{SiO}_{2}\right)_{\mathbf{n}}$ Water* Cement & Slag & Sand \\
\hline OPC & 0 & 0 & 296.8 & 657 & 0 & 1,247 \\
AAS1 & 23.77 & 35.70 & 263.6 & 0 & 604 & 1,247 \\
AAS2 & 5.94 & 95.49 & 234.6 & 0 & 604 & 1,247 \\
AAS3 & 23.77 & 0 & 285.9 & 0 & 604 & 1,247 \\
AAS4 & 47.54 & 0 & 274.8 & 0 & 604 & 1,247
\end{tabular}

${ }^{\star}$ Note: Water content accounts for sand absorption

Table 3. Activating solution properties for AAS mixtures

\begin{tabular}{ccccc}
\hline $\begin{array}{c}\text { Mix } \\
\text { ID }\end{array}$ & $\mathbf{S i O}_{2} / \mathrm{Na}_{2} \mathbf{O}$ & $\mathbf{p H}$ & $\begin{array}{c}\text { Density } \\
(\mathbf{g} / \mathbf{c c})\end{array}$ & $\begin{array}{c}\text { WRA } \\
(\mathbf{c c} / \mathbf{k g})\end{array}$ \\
\hline AAS1 & 0.41 & 14.31 & 1.09 & 5.00 \\
AAS2 & 1.22 & 13.78 & 1.13 & 2.00 \\
AAS3 & 0 & 14.30 & 1.04 & 4.15 \\
AAS4 & 0 & 14.60 & 1.09 & 8.00 \\
\hline
\end{tabular}

\subsection{Sample preparation}


To evaluate shrinkage deformation, a series of "mini-bar" prism samples $(1.27 \times 1.27 \times 12.7 \mathrm{~cm})$ was cast in this study. The smaller dimension of the samples allowed the systems to reach equilibrium in a relatively short period of time. A PVC mold with a gage length of four inches was used in samples preparation. All samples were dried in nitrogen purged Vena VC-10 environmental chambers.

\subsection{Testing procedure}

In order to evaluate the effect of drying rate on shrinkage behavior of AAS, two types of experimental procedures were utilized, namely rapid drying and step-wise drying. Rapid drying involved measuring shrinkage at constant $\mathrm{RH}$ until equilibrium was reached, while step-wise drying measured shrinkage across a broad range of $\mathrm{RH}$. In particular, environmental chambers were programmed for four different humidities $(85 \%, 70 \%, 50 \%$, and $30 \% \mathrm{RH})$ at constant temperature $\left(23 \pm 0.5^{\circ} \mathrm{C}\right)$. The rapid drying shrinkage was measured for all five mixtures (four samples per mixture) at each constant relative humidity under dry nitrogen $\left(\mathrm{N}_{2}\right)$ purge, after seven days of moist curing $(100 \%$ $\left.\mathrm{RH}, 23 \pm 0.5^{\circ} \mathrm{C}\right)$. The concentration of $\mathrm{CO}_{2}$ in environmental chambers was periodically measured with a portable $\mathrm{CO}_{2}$ analyzer and was found to be $0 \mathrm{ppm}$. In case of step-wise drying procedure, the specimens were dried initially at $85 \% \mathrm{RH}$ until they reached equilibrium, at which point the humidity was dropped to $70 \%$. Subsequent $\mathrm{RH}$ reductions to $50 \%$ and $30 \% \mathrm{RH}$ occurred after specimens came to equilibrium at each RH step. Length measurements were taken via a modified digital comparator with an invar reference bar. The accuracy of length measurements was 0.0001 inch. Simultaneous mass measurements were taken using a high precision balance with an accuracy of $0.01 \mathrm{~g}$.

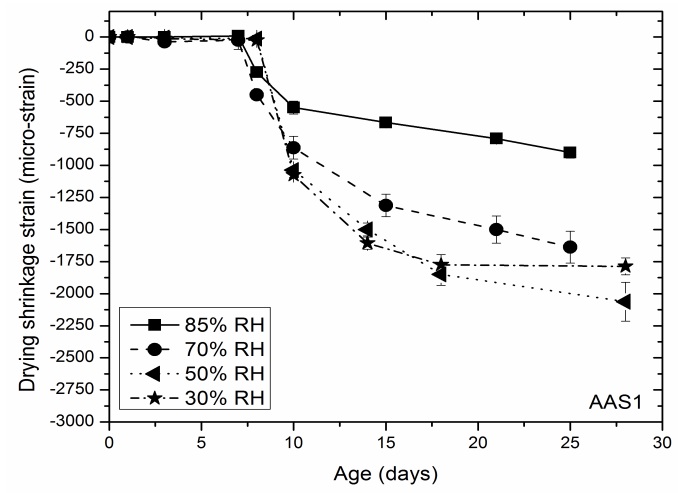

(a)

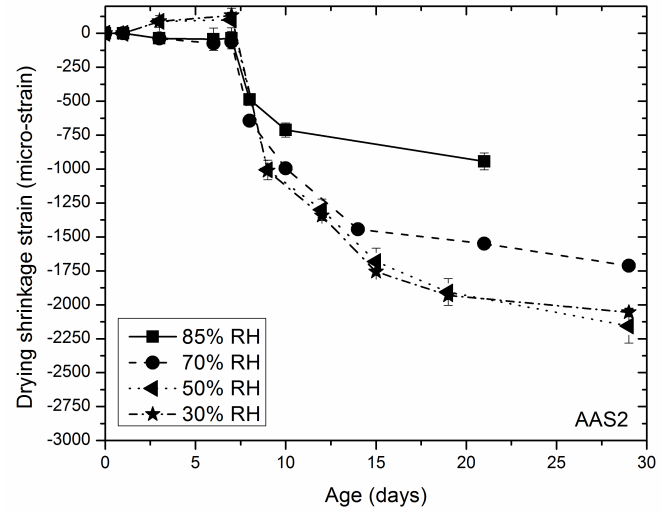

(b)

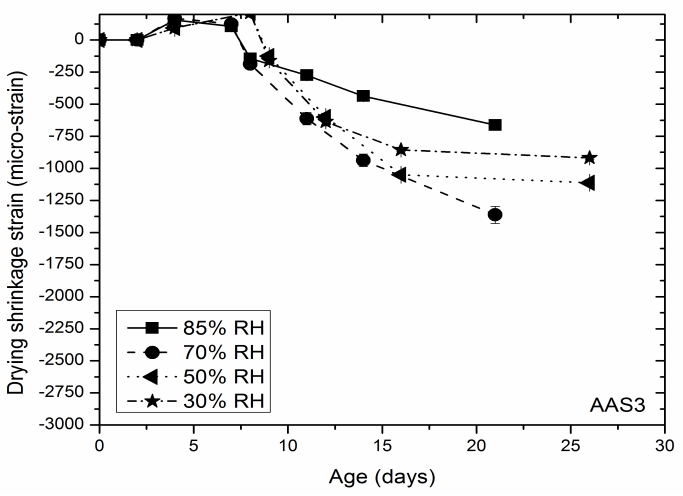

(c)

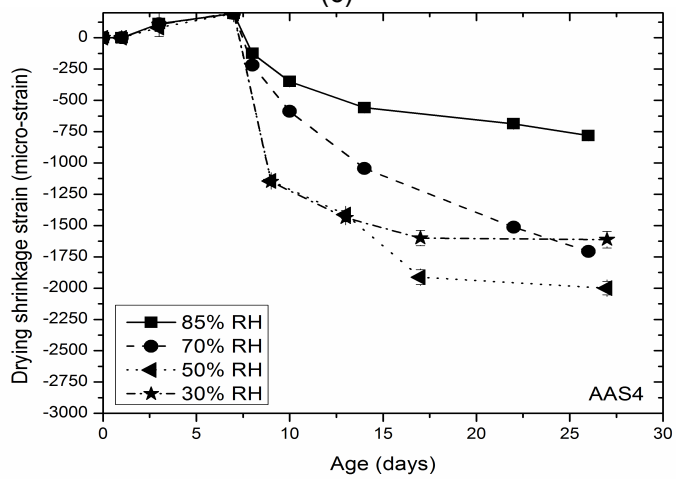

(d)

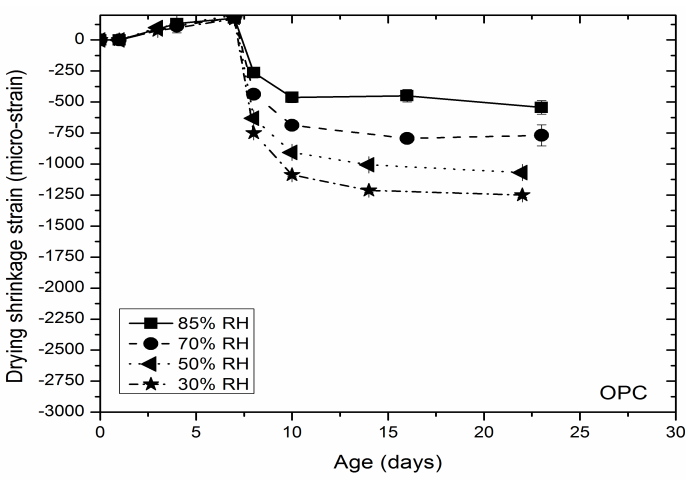

(e)

Figure 1. Rapid drying shrinkage under nitrogen purge for all mortar mixtures (a) AAS1; (b) AAS2; (c) AAS3; (d) AAS4; (e) OPC (after 7 days moist curing) 


\section{EXPERIMENTAL RESULTS}

\subsection{Rapid drying shrinkage of AAS}

Figure 1 shows the results of the measured rapid drying shrinkage of all five mortar mixtures during the first 30 days of testing. All AAS mixtures show higher drying shrinkage strains than the control OPC mixture at all relative humidities, except for AAS3 at 30\% RH.

OPC shows increasing shrinkage with a decreasing relative humidity, which is consistent with the findings of other authors (BaroghelBouny et al., 2006; Hansen, 1987). On the contrary, the AAS mixtures show more complicated drying shrinkage behavior, where the largest ultimate drying shrinkage of AAS3 and AAS4 is observed at $70 \% \mathrm{RH}$. Similarly, AAS1 and AAS2 shrink the most at $50 \% \mathrm{RH}$, even though all mixtures were also dried to $30 \%$ $\mathrm{RH}$.

Figure 2 presents the mass loss data for the four AAS and the control OPC mixtures. It can be seen that for all mixtures, a lower $\mathrm{RH}$ promotes higher moisture loss. Furthermore, moisture loss of each AAS mixture is larger than that for OPC. This contradicts the findings of Collins and Sanjayan, who measured lower mass loss upon drying for AAS than OPC (Collins and Sanjayan, 2000). At each relative humidity, AAS3 showed the highest mass loss. This is likely due to the coarse porosity of the paste in AAS3. The moisture loss for the other three AAS mixtures was measured to be similar.

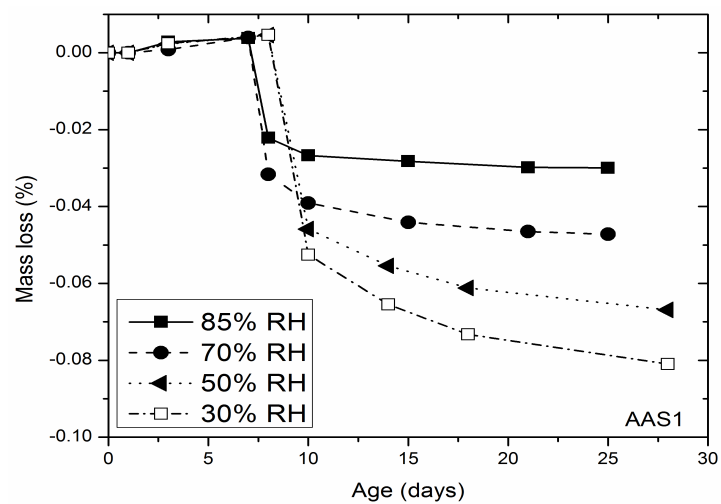

(a)

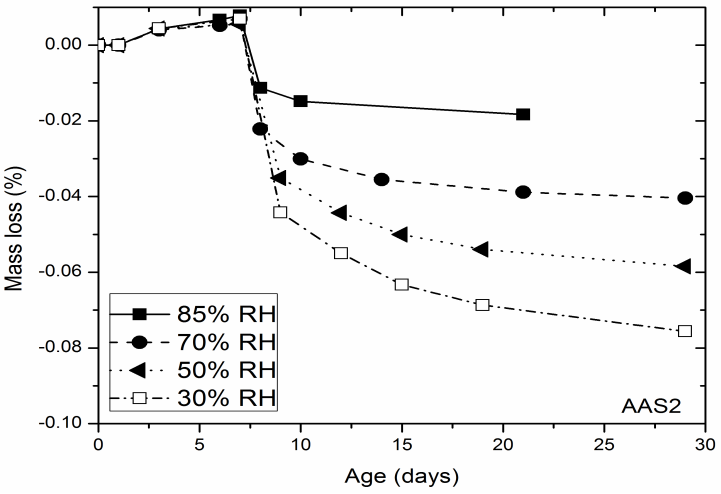

(b)

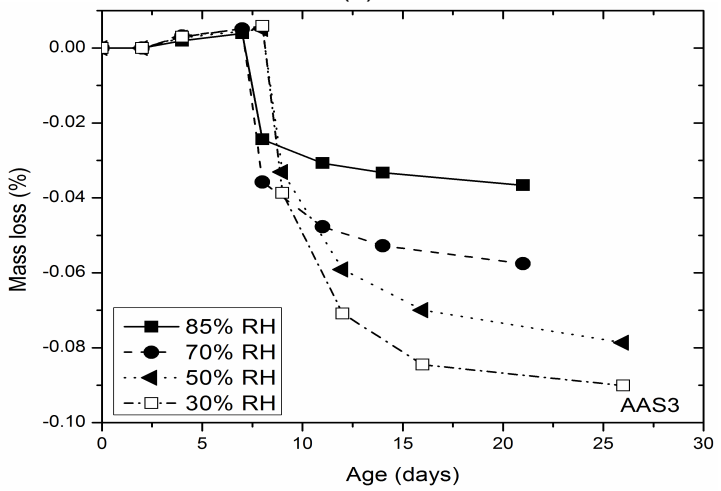

(c)

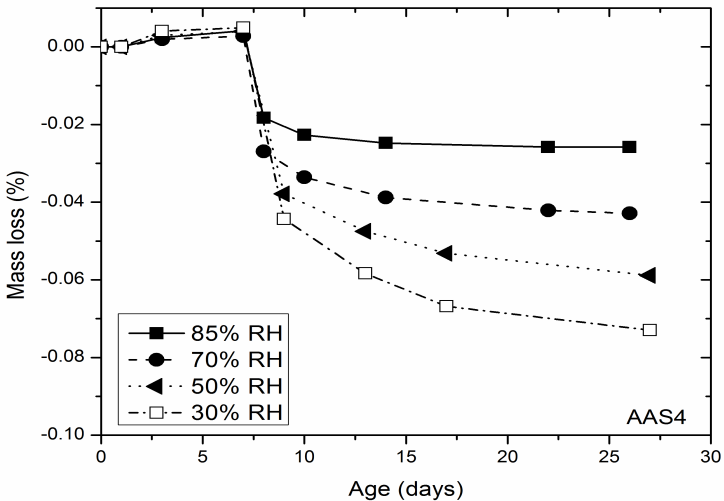

(d)

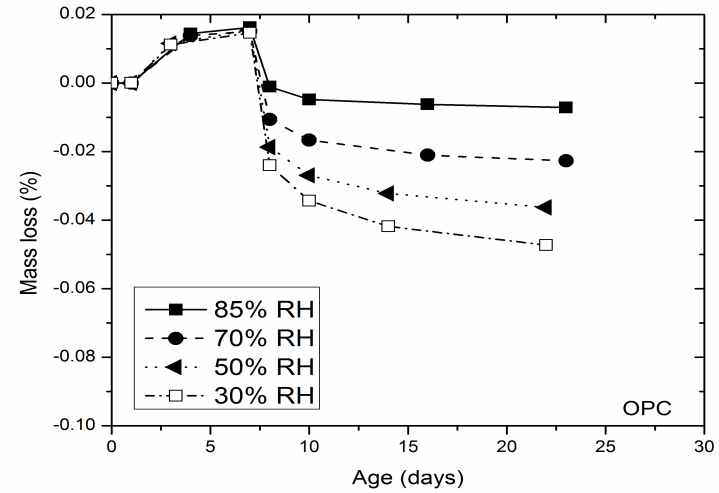

(e)

Figure 2. Mass loss under rapid drying (nitrogen purged) for all mortar mixtures: (a) AAS1; (b) AAS2; (c) AAS3; (d) AAS4; (e) OPC (after 7 days moist curing) 


\subsection{Step-wise drying shrinkage}

The second series of experiments involved drying samples to equilibrium and then gradually dropping the $\mathrm{RH}$. Figure 3 and Figure 4 display the step-wise drying shrinkage and mass loss results of all five mortar mixtures. Similar to rapid drying, the control OPC showed the lowest drying shrinkage and mass loss among all mixtures evaluated. It can be seen that when dried gradually, the relationship between shrinkage and relative humidity for AAS materials differs significantly from that observed under rapid drying. Generally, drying shrinkage of all AAS samples exposed to step-wise procedure showed relatively higher values than those measured in the case of rapid drying. It should be noted here, that ultimately all samples were dried until they reached equilibrium at $30 \%$ $\mathrm{RH}$. Comparing rapid and step-wise drying of AAS3, it can be seen that when dried gradually to a relative humidity of $50 \%$, AAS 3 shows $77 \%$ higher shrinkage deformations that when dried rapidly to the same $\mathrm{RH}$. Similar tendencies are observed for the other AAS mixtures. All mixtures had slightly lower mass loss during the step-wise drying compared to the direct drying process. As such, it can be concluded that drying shrinkage behavior of AAS materials is highly dependent on the rate of drying.

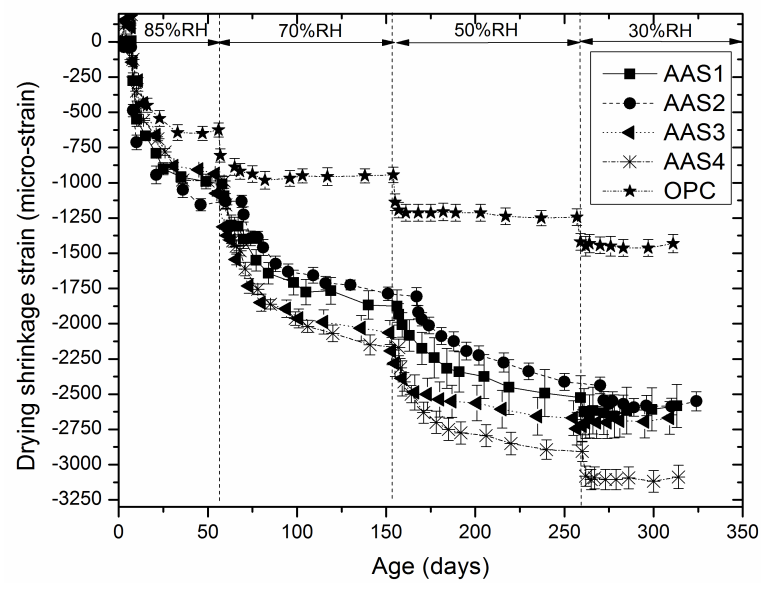

Figure 3. Step-wise drying shrinkage results for all five mortar mixtures.

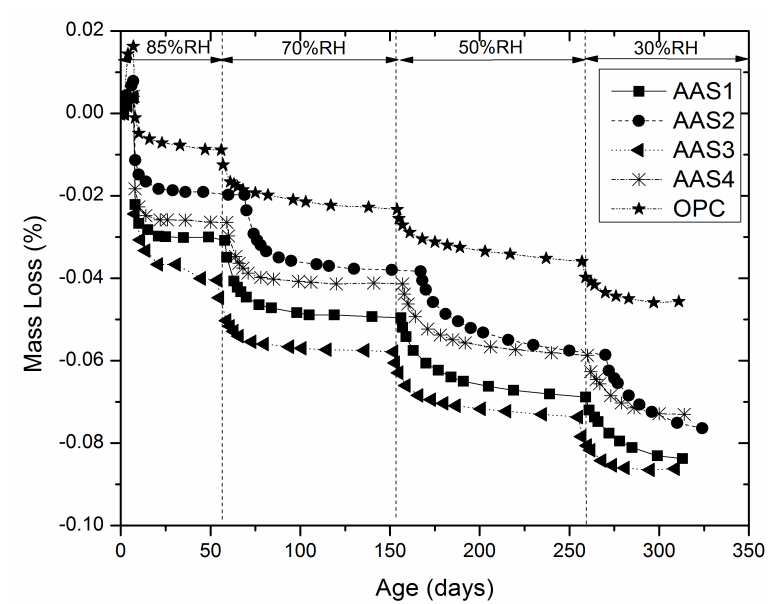

Figure 4. Step-wise drying moisture loss results for all five mortar mixtures.

\section{DISCUSSION}

According to Mackenzie-Bentz equation (Bentz et al., 1998), one can calculate the ultimate paste shrinkage due to capillary stresses $\left(P_{\text {cap }}\right)$ induced by drying:

$$
\varepsilon_{p}=\frac{S}{3} P_{c a p}\left[\frac{1}{K}-\frac{1}{K_{s}}\right]
$$

Where $S$ corresponds to the volumetric degree of saturation, $\mathrm{K}$ is the bulk elastic modulus of the paste (as shown in Table 4), and $K_{s}$ is the bulk modulus of the solid skeleton (e.g., C-S-H or unreacted solids). It can be seen that the material parameters controlling the calculated strain include the degree of saturation, effective bulk modulus and skeleton modulus (Bentz et al., 1998; Cartwright et al., 2014). Capillary pressure is similar at the same $\mathrm{RH}$ according to Kelvin equation (Eq. 2).

$$
P_{\text {cap }}=-\rho_{l} \frac{R T}{M_{l}} \ln (R H)
$$

Where $P_{c a p}$ is the capillary pressure, $\rho_{l}$ is density of liquid, $M_{l}$ is molecular mass and assumed to be same with pure water, $R=8.314[\mathrm{~J} /(\mathrm{mol} \mathrm{K})]$ is the universal gas constant, $T$ is temperature [K]. Additionally, a recent study also showed that the skeleton stiffness $\left(K_{s}\right)$ of AAS is similar to that of OPC (Gebregziabiher and Peethamparan, 2013).

A higher shrinkage of AAS may originate from a higher degree of saturation and lower bulk modulus. As shown in Fig. 5, the measured degree of saturation for OPC is slightly higher 
than that of OPC for each $\mathrm{RH}$ at equilibrium. The effective capillary stress (i.e. $S \cdot P_{\text {cap }}$ ) can be calculated and shown in Fig. 6. It should be noted that the capillary stress theory is primarily valid for $\mathrm{RH}>60 \%$ (approximately) as a liquidvapor meniscus cannot form in pores smaller than those that correspond to $\mathrm{RH}<60 \%$. It can be seen in Fig. 6 that the effective capillary stress of OPC is slightly higher than that of AAS. This would eliminate a higher degree of saturation as a primary cause of higher shrinkage of AAS. Thus, the larger drying shrinkage of AAS could result from a lower stiffness. As shown in Table 4, AAS3 and AAS4 mortars have approximately $33 \%$ lower stiffness, but the stiffness values of AAS1 and AAS2 are similar, or even higher to that of OPC. Therefore, the value of stiffness itself may partially explain the higher shrinkage of AAS3 and AAS4, but cannot properly explain the high shrinkage performance of AAS1 and AAS2. Therefore, the capillary pressure theory, at least for AAS1 and AAS2, cannot explain the high shrinkage performance of AAS by itself and its strong dependence on drying rate.

Table 4. Bulk modulus of Elasticity $\mathrm{K}$ of mortars at 28 days Mix ID Bulk Modulus (GPa)

\begin{tabular}{cc}
\hline AAS1 & 11.34 \\
AAS2 & 10.63 \\
AAS3 & 6.96 \\
AAS4 & 7.42 \\
OPC & 9.44 \\
\hline
\end{tabular}

Note: each data point is an average of 2 duplicate cylindrical specimens)

On the other hand, Eq. 1 assumes a linear elastic behavior and does not account, for example, for creep. Creep is a phenomenon that results in the gradual increase in deformation under a constant stress. As shown in Fig. 1 and Fig. 2, the mass loss of AAS plateaus much faster than that of shrinkage. This means that AAS mixtures continue to shrink even though they are not losing any more water. This may suggest significant creep deformation in AAS. While for OPC, the shrinkage practically ceases when moisture loss reaches equilibrium. This is strong evidence that creep plays a significant role in drying shrinkage of AAS.

The correlation between relative humidity and drying shrinkage for all five mortar mixtures subjected to rapid drying procedure has been shown in Fig. 7. It can be seen that the OPC mixture shows a bi-linear relationship, while AAS mixtures show a parabolic relationship between ultimate shrinkage and relative humidity. Particularly, AAS1 and AAS2 showed the highest shrinkage at $70 \% \mathrm{RH}$, while AAS3 and AAS4 showed the highest shrinkage at $50 \% \mathrm{RH}$. This unique phenomenon notably demonstrates the influence of drying rate on shrinkage behaviors of AAS.

Interestingly, when dried under the step-wise process, the shrinkage behavior of AAS was found to differ significantly compared to rapid drying, where the highest drying shrinkage deformations were observed at the lowest relative humidity. This may be due to the formation of permanent shrinkage strains (e.g. creep) during the gradual drying process.

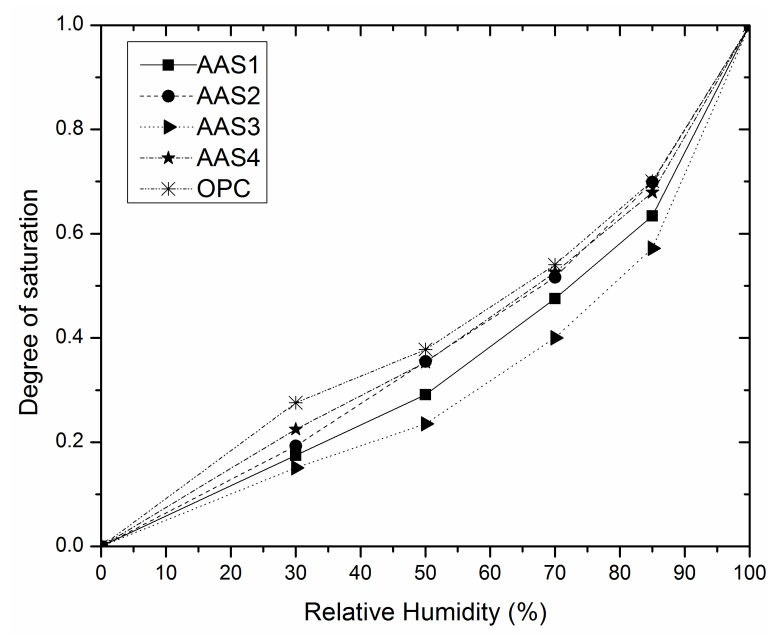

Figure 5. The correlation between relative humidity and degree of saturation at equilibrium for all five mortar mixtures

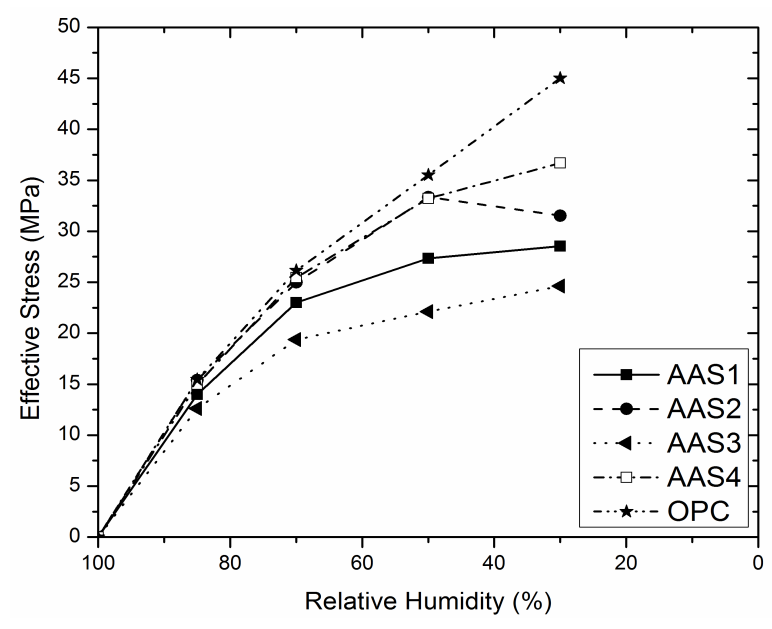

Figure 6 . The correlation between relative humidity and drying shrinkage for all five mortar mixtures 


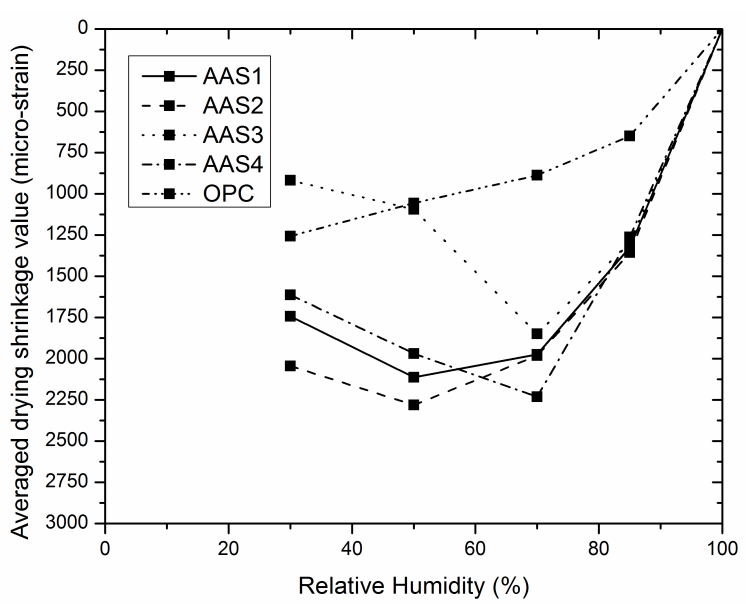

Figure 7. The correlation between relative humidity and drying shrinkage for all five mortar mixtures

It is possible other shrinkage driving forces, (e.g. Gibbs-Bangham strain) play an important role in shrinkage behavior of AAS, since the notably dependent shrinkage behavior of AAS on drying rate cannot be completely explained in present studies. The investigation of the shrinkage mechanisms of AAS is currently a main research focus at Penn State.

\section{CONCLUSIONS}

In this paper, the influence of drying rate on shrinkage characteristics of AAS was studied. In particular, four AAS mortar mixtures and one control OPC mortar were exposed to different drying procedures, namely rapid drying and step-wise drying. For rapid drying, four different relative humidities (i.e. $30 \%, 50 \%, 70 \%$ and $85 \%$ $\mathrm{RHs}$ ) were utilized to investigate the shrinkage behavior of AAS. In step-wise drying scenario, a gradual change of $\mathrm{RH}$ from $85 \%$ to $30 \% \mathrm{RH}$ was applied.

The following conclusions can be drawn according to the experimental results and analysis:

(1) AAS mixtures have a unique parabolic relationship between relative humidity and ultimate drying shrinkage that differs significantly from the bi-linear correlation found in OPC mixtures.

(2) Drying rate significantly affects the drying shrinkage behavior of AAS mortars.

(3) Creep deformation seems to contribute significantly to drying shrinkage of AAS.

(4) Effective capillary stress ( $\left.S \cdot P_{\text {cap }}\right)$ was estimated to be slightly higher for OPC than
AAS and cannot explain the high shrinkage of AAS

(5) The stiffness of mixtures activated by $\mathrm{NaOH}$ is smaller than that of OPC and contributes to their high shrinkage deformations.

\section{ACKNOWLEDGEMENTS}

The authors gratefully acknowledge the financial support from the National Science Foundation (NSF) under Award CMMI \#1265789. Any opinions, findings and conclusions or recommendations expressed in this material are those of the authors and do not necessarily reflect the views of the National Science Foundation. The authors are also appreciative of the invaluable assistance of Mr. Jared Wright and Mr. Dan Fura. All tests were performed at the Civil Infrastructure Testing and Evaluation Laboratory (CITEL) and the Materials Research Institute (MRI) of Penn State.

\section{REFERENCES}

Bakharev, T., Sanjayan, J., Cheng, Y.-B., 2000. Effect of admixtures on properties of alkaliactivated slag concrete. Cement and Concrete Research 30, 1367-1374.

Bakharev, T., Sanjayan, J.G., Cheng, Y.-B., 1999. Alkali activation of Australian slag cements. Cement and Concrete Research 29, 113-120.

Baroghel-Bouny, V., Mounanga, P., Khelidj, A., Loukili, A., Rafaï, N., 2006. Autogenous deformations of cement pastes: part II. W/C effects, micro-macro correlations, and threshold values. Cement and Concrete Research 36, 123-136.

Bentz, D.P., Garboczi, E.J., Quenard, D.A., 1998. Modelling drying shrinkage in reconstructed porous materials: Application to porous Vycor glass. Modelling and Simulation in Materials Science and Engineering 6, 211.

Cartwright, C., Rajabipour, F., Radlińska, A., 2013a. Shrinkage Characteristics of AlkaliActivated Slag Cements, 3rd International Conference on Sustainable Construction Materials and Technology - SCMT2013, Ktoto, Japan.

Cartwright, C., Rajabipour, F., Radlińska, A., 2014. Shrinkage Characteristics of AlkaliActivated Slag Cements. ASCE Journal of Materials in Civil Engineering (accepted).

Cartwright, C.P., Rajabipour, F., Radlińska, A., 2013b. Measuring the Chemical Shrinkage of 
Alkali-Activated Slag Cements Using the Buoyancy Method, Mechanics and Physics of Creep, Shrinkage, and Durability of Concrete: A Tribute to Zdenek P. Bažant: Proceedings of the Ninth International Conference on Creep, Shrinkage, and Durability Mechanics (CONCREEP-9), September 22-25, 2013 Cambridge, Massachusetts. ASCE Publications, p. 308.

Collins, F., Sanjayan, J., 2000. Effect of pore size distribution on drying shrinking of alkaliactivated slag concrete. Cement and Concrete Research 30, 1401-1406.

Gebregziabiher, B.S., Peethamparan, S., 2013. Characterization of Alkali Activated Slag Gel using Nano-indentation, 3rd International Conference on Sustainable Construction Materials and Technology - SCMT2013, Ktoto, Japan.

Hansen, W., 1987. Drying shrinkage mechanisms in Portland cement paste. Journal of the American Ceramic society 70 , 323-328.

Jiang, M., Chen, X., Rajabipour, F., Hendrickson, C.T., 2014. Comparative Life Cycle Assessment of Conventional, Glass Powder, and Alkali-Activated Slag Concrete and Mortar. Journal of Infrastructure Systems.

Melo Neto, A.A., Cincotto, M.A., Repette, W., 2008. Drying and autogenous shrinkage of pastes and mortars with activated slag cement. Cement and Concrete Research 38, 565-574.

Shi, C., Roy, D., Krivenko, P., 2005. Alkaliactivated cements and concretes. Taylor \& Francis. 